\title{
Risks and Opportunities for Reforming Construction with Blockchain: Bibliometric Study
}

\author{
Mohammad Darabseh ${ }^{\text {a* }}$, João Poças Martins ${ }^{b}$ \\ ${ }^{a}$ Faculty of Engineering (FEUP), University of Porto, Rua Dr. Roberto Frias s/n, 4200-465 Porto, Portugal. \\ ${ }^{b}$ CONSTRUCT - GEQUALTEC, Faculty of Engineering (FEUP), University of Porto, Rua Dr. Roberto Frias s/n, 4200-465 Porto, Portugal.
}

Received 10 February 2020; Accepted 30 April 2020

\begin{abstract}
Emerging technologies are always treated with caution, and Blockchain is no exception to this rule. The AECO (Architecture, Engineering, Construction, and Operations) sector is considered conservative when it comes to technology adoption, which is often positive in an industry that deals with a vital commodity that could harm humans or result in catastrophe. However, excessive caution also stifles innovation, as shown in the industry's low R\&D expenditure when compared to other industrial activities. In recent years, the AECO sector has benefited from the adoption of new information management tools and systems that allow professionals to develop, share and store construction data more effectively. These tools have successfully tackled many of the traditional interoperability issues that have affected the industry, but several challenges remain unsolved. Indeed, effective real-world communication depends, not only on the compatibility between data formats and systems, but also on issues such as privacy, transparency, and trust. Blockchain has been adopted in different activities as a tool to address these issues, but its impact in the construction industry remains scarce. This paper presents a bibliometric study for the available literature on Blockchain implementation in the AECO sector. In addition to the bibliometric review, content analysis for the literature retrieved is presented to provide a clear vision of the current directions regarding Blockchain technology adoption. The purpose of this article is to evaluate the maturity of Blockchain in Construction literature using statistics based on the available bibliometrics in addition to content analysis. The main results show that although the number of articles about the use of Blockchain in Construction has increased, no studies that present ready to use solutions were found. Instead, the covered studies discuss the technical capabilities of the technology and suggest possible fields of implementation, such as smart contracts and automated payments. Study limitations include the limited amount of literature that can be found on major indexing services, which cover a relatively short reference period.
\end{abstract}

Keywords: Blockchain; Construction; Bibliometric Review; BIM; Construction Management; Infrastructure.

\section{Introduction}

Blockchain, as a concept, is not new. However, it gained its current popularity after it was used to create the first cryptocurrency in January 2009, Bitcoin. Crosby et al. (2016) [1] defines Blockchain as a distributed database of records, or public ledger of all transactions or digital events that have been executed and shared among participating parties. Blockchain is essentially a protected distributed file system where communication between nodes is not coordinated by a single node. In Blockchain, data integrity can be assured through different consensus mechanisms such as proof of authority, proof of space, proof of stake, or proof of work. Blockchain brings value and transparency to the data exchange network and makes collaboration efficient [2].

* Corresponding author: darabseh@outlook.com

http://dx.doi.org/10.28991/cej-2020-03091541

(C) 2020 by the authors. Licensee C.E.J, Tehran, Iran. This article is an open access article distributed under the terms and conditions of the Creative Commons Attribution (CC-BY) license (http://creativecommons.org/licenses/by/4.0/). 
The body of literature on this topic includes different types of studies such as research articles or review articles, starting from 2017. This study uses two methods of literature examination: (1) Bibliometrics by using the literature citations and meta data to provide the reader with general research directions, research groups and gaps from the literature; (2) Content analysis where literature gathered from different sources are examined then categorized according to their content. Bibliometrics and content analysis are a powerful combination to identify current research trends, and to benchmark progress within a field during a period of time.

This article reviews the existing literature on the use of Blockchain in the construction industry. The two main goals of this study are the following: (1) to contribute to the available body of literature, which is currently limited due to the short time span for researchers interested in implementing the technology within the AEC industry; (2) to assess the existing literature and research trends by using bibliometrics.

This article is structured as follows: after this Introduction chapter, section two presents the research methodology, chapter three presents the bibliometric analysis. The content analysis is presented in section four, where the articles were categorised into subtopics according to their main contents. The conclusions section includes a summary of the work performed and future expectations while considering the research limitations.

\section{Methodology}

\subsection{Paper Retrieval and Bibliometric Analysis}

The literature, including journal articles and conference papers and books, were retrieved from two sources Web of Knowledge and Scopus databases on Wednesday, 23/10/2019. For both databases, the same search string was used: Blockchain AND construction; The filtering option adopted in Scopus was (Article title, Abstract, Keywords), and for Web of Knowledge the filter option was (Topic). The primary search resulted in 190 records in Scopus and 70 records in Web of Knowledge. After the filtration process detailed in Table 1, the total number of records considered for this review is 40 records, including 20 journal articles, 19 conference papers, and one book chapter.

Table 1. Paper retrieval and filtration process

\begin{tabular}{ccc}
\hline Process/ Database & Scopus & Web of Knowledge \\
\hline Primary search with (Blockchain AND construction) & 190 records & 70 records \\
Filter by English only & 182 records & 69 records \\
Filter by title and abstract & 32 records & 48 records \\
Total retrieved records & 40 records \\
Total records after removing duplicates & & \\
\hline
\end{tabular}

The software used to extract the bibliometric results is the data manipulation software $\mathrm{R}, \mathrm{R}$ studio distribution, using the BIBLIOMETRIX package. The harvested data from both databases was imported to the software and then processed and merged using BIBLIOMETRIX library functions. The software package was then used to extract the following bibliometric information from the provided data: (1) annual scientific production; (2) authors coupling; (3) average article citation per year; (4) average total citations per year; (5) co-citations network; (6)conceptual structure map; (7) country collaborations; (8) factorial map of most cited documents; (9) factorial map of the documents with the highest contributes; (10) keywords co-occurrences; (11) most productive authors; (12) most productive countries [3]. Not all the extracted information is analysed in this article due to the short time span of the assessment and to its small sample size. Figure 1 shows the workflow to extract bibliometric results using R studio.

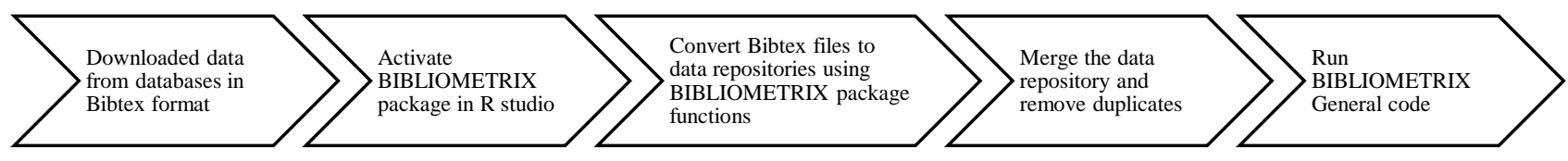

Figure 1. Bibliometrics extraction process using BIBLIOMETRIX package

\subsection{Content Analysis}

The 40 articles selected for this were analysed to categorised according to the theme they cover. Three categories were identified: construction management, BIM and civil engineering practices and Infrastructure. However, BIM is a 
dominant topic in all the literature which is expected because BIM currently considered the change catalyst for construction data improvement.

\section{Bibliometric Analysis}

\subsection{Time Series Analysis}

Table 2 shows the chronological distribution of the available literature, which starts from 2017 to 2019, with a total of 40 papers. The primarily conclusion that can be drawn is that Blockchain in construction is a very recent research topic, with increasing interest during its short lifetime.

Table 2. Annual scientific production

\begin{tabular}{cc}
\hline Year & Articles \\
\hline 2017 & 2 \\
2018 & 8 \\
2019 & 30 \\
\hline Total & 40 \\
\hline
\end{tabular}

\subsection{Keyword Analysis}

A co-occurrence analysis for keywords was performed for the 40 selected articles. Figure 2 shows the results as a colour-coded network of word groups. The size of each node is proportional to its frequency, and the connecting line between two nodes indicates the strength of the link between them. The connection between Blockchain and BIM is notable, and this will be elaborated in the content analysis. Three categories were identified from the co-occurrence analysis for keywords: (1) construction management terms, including project management and construction contract; (2) design-related terms, such as building information modelling; (3) other emerging technologies such as big data, internet of things. The analysis shows the most common co-occurrence keywords are: Blockchain 26 times, BIM 12 times and smart contracts seven times.

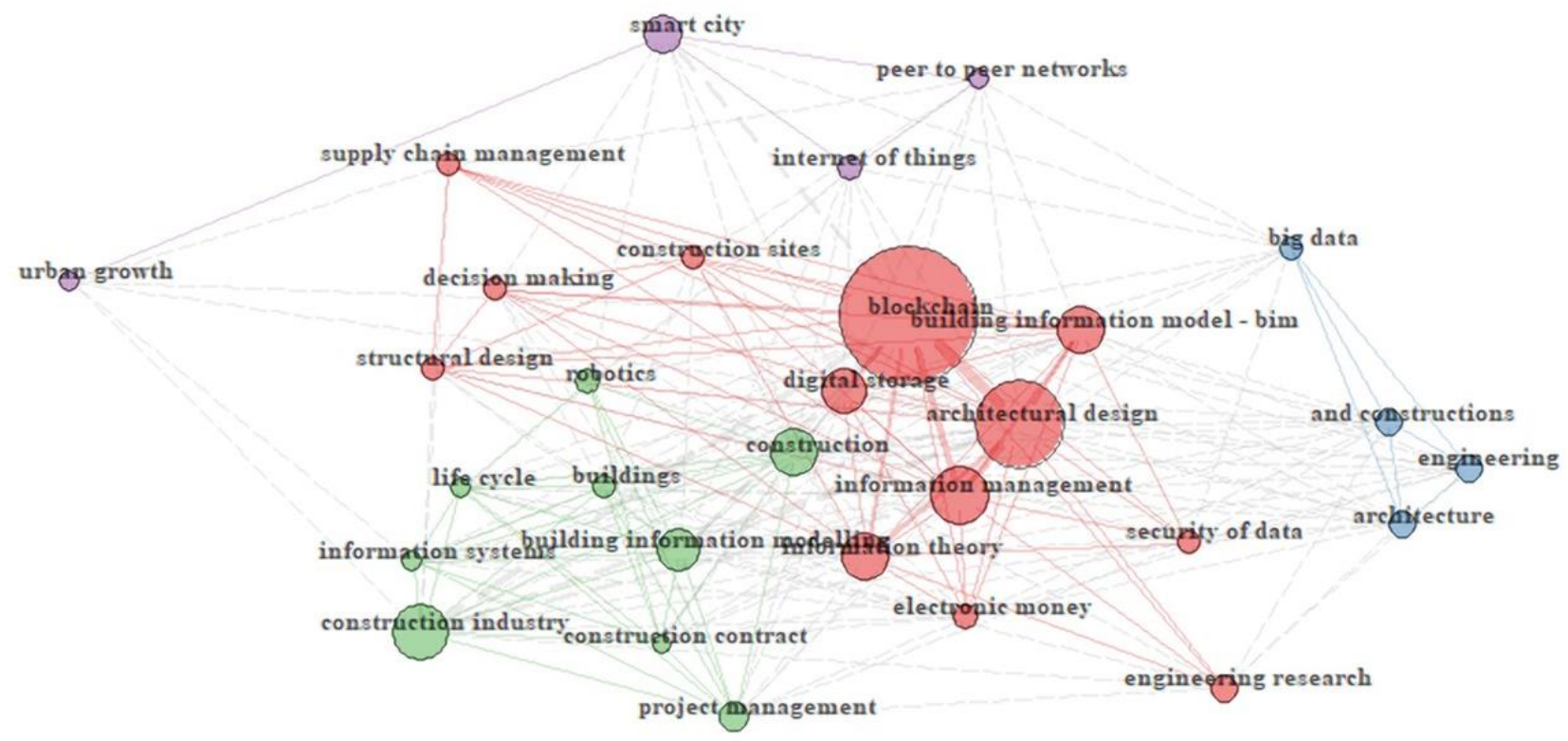

Figure 2. Words co-occurrence network

\subsection{Top Cited Articles}

The 34 articles citation analysis shows that Turk and Klinc [4] is the most cited article with a total citation of 28 and with total citation per year of 14 . This is somewhat expectable, as the author himself mentions that no results were found in the literature for the combination of keywords construction and Blockchain at the time of writing. Table 3 shows the top manuscripts per citations. 
Table 3. Top manuscripts per citations (TC: Total Citations)

\begin{tabular}{ccclcc}
\hline Rank & Author & Year & Source & TC & TC/per year \\
\hline 1 & TURK & 2017 & PROCEDIA ENG & 28 & 14.0 \\
2 & WANG J & 2017 & FRONT ENG MANAG & 17 & 8.5 \\
3 & LI S & 2018 & PROC - IEEE INT CONF SMART INTERNET THINGS, & 7 & 7.0 \\
4 & LANKO A & 2018 & MATEC WEB CONF & 4 & 4.0 \\
5 & JO BW & 2018 & SENSORS & 3 & 3.0 \\
6 & LI J & 2019 & AUTOM CONSTR & 3 & - \\
7 & NAWARI NO & 2019 & J BUILD ENG & 2 & - \\
8 & NAWARI NO & 2019 & J INF TECHNOL CONSTR & 2 & \\
9 & YE Z & 2018 & ISARC - INT SYMP AUTOM ROBOT IN CONSTR INT & 2 & 2.0 \\
10 & ABLYAZOV T & 2019 & IOP CONF SER MATER SCI ENG & 1 & - \\
\hline
\end{tabular}

\subsection{Most Productive Authors and Most Productive Countries}

As expected, the results on the most productive authors and countries are non-conclusive because of the limited amount of literature and the short time span. However, by numbers, the most productive authors are Nawari and Ravindran, who co-authored three articles, and the most productive country was China with four articles. Figure 3 shows the most productive authors, and Figure 4 shows the most productive countries.

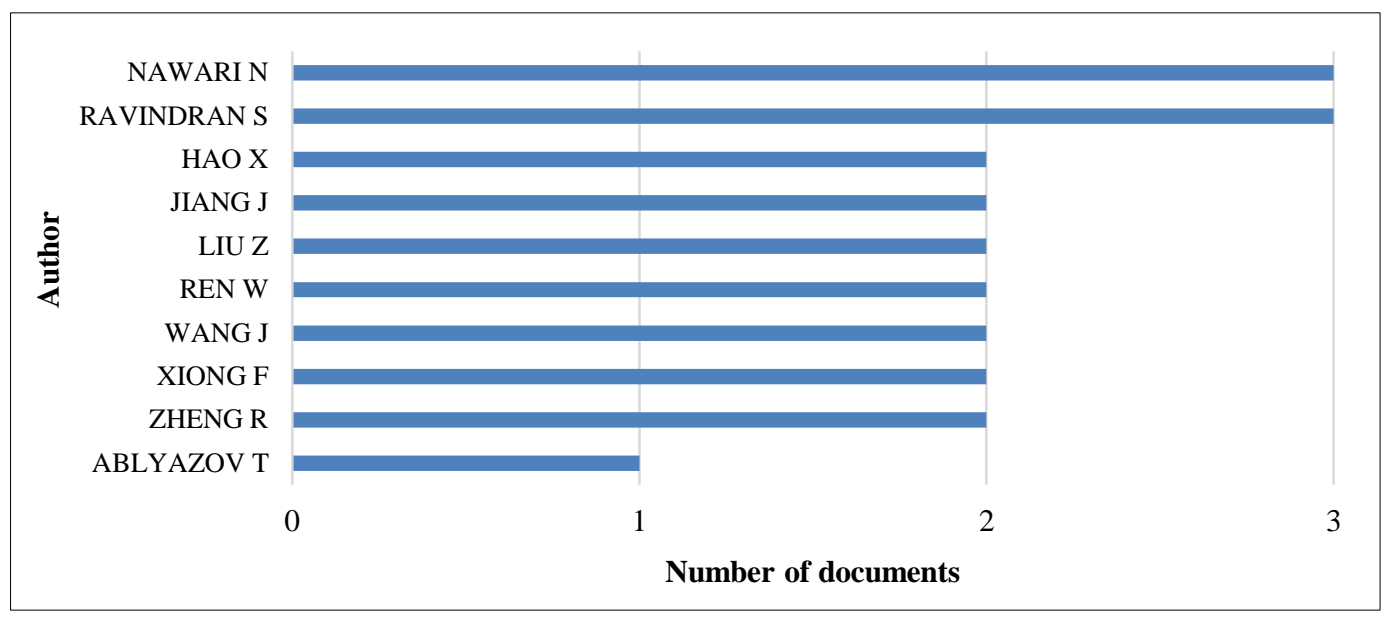

Figure 3. Most productive authors

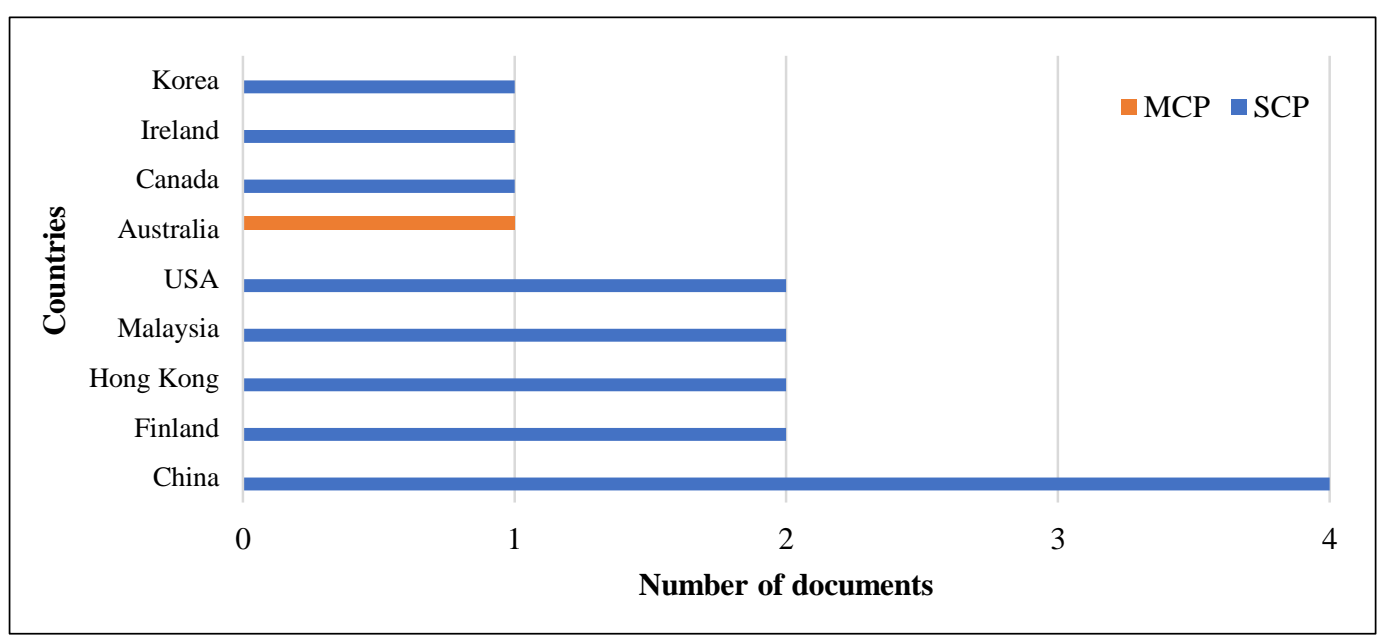

Figure 4. Most productive countries (SCP: single country publications, MCP: Multiple country Publications) 


\subsection{Co-authorship Analysis}

The coupling analysis shows three groups of researchers, as presented in Table 4. The colour-coded author's coupling plot is shown in Figure 5, where higher lines density represents increased collaboration.

Table 4. Identified research groups

\begin{tabular}{cl}
\hline Researcher group & \multicolumn{1}{c}{ Participants } \\
\hline 1 & Nawari O. Nawari and Shriraam Ravindran \\
2 & Rongyue Zheng, Jianlin Jiang, Xiaohan Hao, Wei Ren, Feng Xiong, and Tianqing Zhu \\
3 & Jennifer Li, David Greenwood, Mohamad Kassem \\
\hline
\end{tabular}

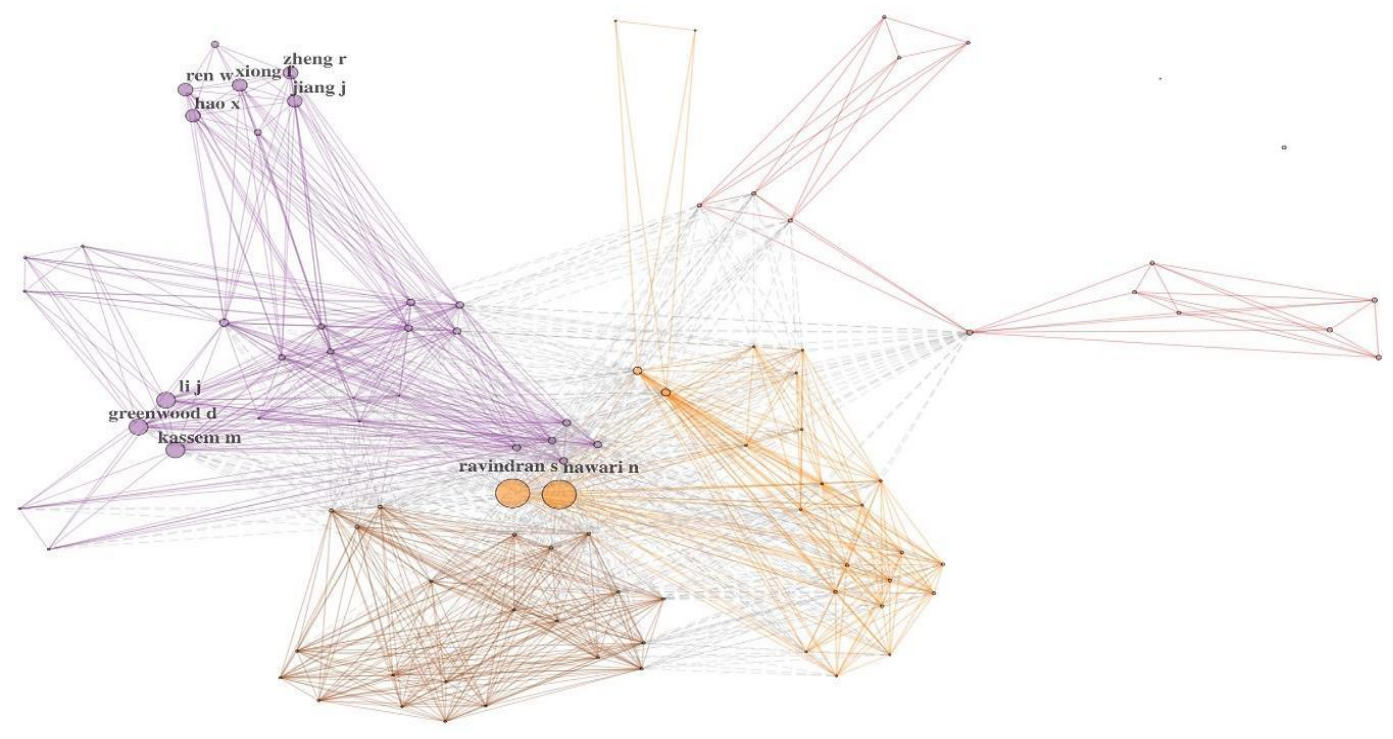

Figure 5. Identified work groups in the literature

\section{Content Analysis}

To provide a deeper understanding of the collected literature, a content analysis was performed. The technology itself is introduced first; then, the literature is classified according to the main topic covered. The topics include construction management, BIM, construction data, supply chain, concrete quality, permits compliance and smart cities.

\subsection{Blockchain as a Technology}

From a general point of view, Blockchain is a communication protection protocol that protects data from manipulation from unauthorised parties. However, implementing Blockchain can take different forms according to the data type and source. For example, implementing Blockchain for architectural design data is different from implementing it for the construction payment process. This article will investigate the proposed applications, the expected results of implementing it, the benefits, the barriers and risks that hamper the implementation process from the researchers' points of view. However, as this topic is a combination of information technology and civil engineering, the content analysis focus will be on the technology applications in construction without technical details about the technology itself unless necessary.

\subsection{Blockchain and Construction}

Chronologically, Turk and Klinc [4] was the first author to publish an article that combines the keywords Blockchain and construction, which explains having the highest number of citations between records. Turk and Klinc [4] work was an investigation aiming to link the Blockchain features to the existing problems facing construction management processes. Turk and Klinc [4] stated that Blockchain in construction would be different from bitcoin Blockchain in three specifications: (1) number of transactions; (2) number of participants; (3) size of data exchanged. A comparison between the expected construction Blockchain and current Bitcoin Blockchain, according to Turk, is shown in Table 5. 
Table 5. Turk and Klinc [4] comparison between Bitcoin Blockchain and construction Blockchain

\begin{tabular}{cccc}
\hline Specifications & Number of transactions & Number of participants & Data size \\
\hline Bitcoin Blockchain & billions & millions & kilobytes \\
Construction Blockchain & hundreds & dozens & gigabytes \\
\hline
\end{tabular}

Hardin and McCool [5] argue that successful BIM requires three components: (1) tools; (2) behaviours and (3) processes where he explains that companies tend to use new technologies with old processes. Therefore Turk and Klinc [4] predicts that BIM and Blockchain should have an inseparable future for companies who want to use BIM efficiently, especially for document management purposes. Blockchain will help with managing digital intellectual property and provide the tool needed to address the legal accountability issue in BIM-based projects. Blockchain will improve inter-organizational traceability and provide a non-centralised data ownership record for the industry [4]. Turk summarises Blockchain features in three points: (1) the content is chained in many devices; (2) the chained data cannot be modified; (3) the copies in multiple sources are identical. These features can be used in the construction industry to have an immutable decentralised comprehensive register that contains the entire data with its metadata, which will eliminate the need for a central authority. Turk concludes that BIM was a good start for the construction industry to move from generic business solutions to solutions that are customised for the construction industry needs. However, Blockchain is required to resolve current BIM problems such as data ownership and changes tracing.

Wang et al. [6] followed Turk and fitted construction industry applications in between Blockchain technology generations 2.0 and 3.0. Blockchain 2.0 focuses on market decentralisation and asset transfer. Blockchain 3.0 focuses on legal applications such as governmental Blockchain applications [6]. Blockchain provides solutions for Notarization applications to verify documents authenticity, transaction applications like payments, procurement tools, and provenance applications to improve construction processes transparency and traceability. Identified challenges for using Blockchain according to Wang et al. [6] are (1) technical challenges: which arise from Blockchain itself as new technology; (2) construction business challenges: convincing companies to switch from in-house records to a permissioned distributed ledger will be a hard job especially for companies who have invested heavily in building their own Enterprise Resources Planning (ERP) systems; (3) human-related challenges: low-level of awareness and understanding for emerging technology is an expected challenge that prevents the technology from expanding.

Shojaei [7] sees construction benefiting from Blockchain in the following applications: (1) smart contracts, (2) supply chain management and circular economy; (3) Building Information Modelling (BIM); (4) Facility Management (FM); (5) sustainability. The term smart contract was first used by Nick Szabo in 1994 when he proposed using digital contracts as a guarantee for automated execution when requirements were met [8]. After the Bitcoin boom, smart contracts reappear with the increased interest in Blockchain. The adoption of smart contracts in construction can transform the way projects' Work Breakdown Structures (WBS) are developed. New WBS will be Smart WBS (SWBS), which are built as small packages accompanied by obligations, requirements, liabilities updated in real-time. Besides smart contracts, the construction supply chain can benefit from Blockchain by replacing the bureaucratic supply ordering to a seamless process where inventory can be checked, priced, ordered, paid and tracked without the need for third-party involvement. A circular economy based on Blockchain requires product profiles to come with extensive detailed information containing the raw material sources and characteristics. Processes such as maintenance or recycling will thus be facilitated and become more manageable. As for BIM and FM, the author considers BIM plus Blockchain will be an effective combination of tools to control data changes and improve collaboration. FM using Building Maintenance Systems (BMS) plus Blockchain is also expected to help controlling the advanced processes such as maintenance and demolition. The last field discussed by Shojaei [7] was sustainability. Sustainability goals will be easier to achieve if Blockchain was used in the supply chain because of material transparency which will give accurate whole life cycle cost and carbon emission estimates. The author concludes by suggesting that construction software developers make it possible to pull data from Blockchain databases.

San et al. [9] claim that Blockchain has features which are needed in today's construction industry, such as (1) decentralisation; (2) autonomy; (3) peer-to-peer relationship; (4) record immutability; (5) time-stamping. These features can be used in five construction-related practices: (1) contract management; (2) Electronic Document Management (EDM); (3) BIM; (4) property management; (5) supply chain management. Consequently, the author believes that it will improve construction project workflow, time and cost utilisation, transparency and trust among project participants, construction projects data security, collaboration in international construction [9].

The current paucity of available case studies in Blockchain applications in construction requires researchers to search for suitable use-cases from other activities instead. Hargaden et al. [10] mention case studies from related industries where Blockchain is used to reduce bureaucracy, reduce counterfeiting and provide traceability. One case study found in the construction industry was Bimchain, which aims to make BIM collaborative and function as a litigation solving and legally binding tool [10]. Luo et al. [11] suggest a payment automation system built on a permissioned-Blockchain. Delay in construction payments is a serious problem that can lead to companies' 
bankruptcy, especially for small contractors. Reasons for delay in construction payment vary; however, many of these reasons are a result of the relationships among stakeholders are adversarial and hostile in addition to the complex and uncertain nature of construction projects. Payment automation is an effective solution to this problem. The proposed solution contains two ways for executing payment: (1) automatic, through smart contracts; (2) manually, requiring action to be taken by the stakeholder. This proposal depends on integrating BIM and Blockchain because requirements are first added to BIM files then broadcasted to the Blockchain network [11].

The issue of trust between stakeholders, or the lack thereof, is the main issue discussed by Goh et al. [12], who asserts that Blockchain shifts the need to trust a person or an entity to a transparent data exchange protocol. The authors claim that Blockchain has the potential to facilitate automating repetitive tasks and to reduce rework and items recall, which is expected to result in overall cost reduction in construction projects. The authors also present considerable challenges to the adoption of Blockchain in Construction. These challenges include technical obstacles, such as hardware and software development, which require companies to invest in their technological infrastructure while the uncertainty about Blockchain is still significant.

Graham and Hailer [13] investigated the current level of Blockchain adoption in the construction industry by examining two software companies that provide solutions based on this technology, Brickschain and Intelliwave Technologies' SiteSense. The authors conclude that even though Blockchain has the potential to solve serious problems in the construction industry such as poor data coordination, rework and litigations, there is a persistent need for further research regarding the technology implementation and development cost.

\subsection{Blockchain and BIM}

BIM and Blockchain are often co-occurring because Blockchain handles data, and BIM is the primary source of data in modern construction.

Safa et al. present Blockchain as a solution for common industry problems, such as trust and collaboration. Safa et al. claim that the consortium-chain model fits the industry needs better than strictly private or public models. Indeed, the number of nodes in consortium blockchains is not small enough to have the possibility of $51 \%$ attack or controlled like private Blockchain. Unlike public Blockchains, the number of nodes is limited, which limits the risks of posting sensitive documents on the network. These authors also predict that project complexity will be reduced because Blockchain will facilitate management and communication tasks during the project's lifecycle. A special relationship is expected between BIM and Blockchain, which can be combined to address the following problems: (1) document ownership, (2) copyright protection, (3) modifications, and error responsibilities [14].

Nawari and Ravindran [15] suggest that several challenges introduced by BIM could be overcome if Blockchain is implemented as a data management and protection tool. The authors claim that BIM lacks effective tools for the Request for Information (RFI) management process, versioning and archival management. Also, real-time tracking during design and construction is still inefficient [15].

Liu et al. (2019) classify BIM challenges in the design process as follows: (1) BIM adopting threats including: technical, financial, and legal threats; (2) digital content rights in addition to cyber threats; (3) model reuse policies; (4) blind trust in the team members in exchange of collaboration; (5) BIM contracts litigations. The authors expect several improvements for sustainable design systems when Design is integrated with Blockchain [16].

The traditional BIM model file contains the last saved version without a modification record. Even if a tracking tool were used, it would be hard to ensure the validity of the information and that it was not tampered by the network operators. Zheng et al. [17] propose a framework (named BcBIM) to make BIM data provenance and accountability achievable. BcBIM is a combination of BIM and consortium Blockchain technology to provide a comprehensive, timestamped record of modifications in a BIM model. BcBIM uses BIM as a service (BIMaaS) to outsource BIM data storage and computation which will make models accessible from mobile terminals. Bukunova and Bukunov [18] consider BIM as a solution for many of the industry' s long-term problems, such as long design period, quality and transparency, efficiency, and flexibility in construction projects. Nevertheless, BIM produces challenges of its own, including data segmentation, overlapping and poor exchanging mechanisms. These interoperability issues could benefit from Blockchain to provide efficient copyrights management for technologies and protocols embedded inside the software to encourage developers to work together by protecting their intellectual property rights.

The combination of three technologies BIM, IoT, and Blockchain, was presented as the "cup-of-water theory" by Ye et al. [19]. According to the authors, all three components are compulsory in this system for the construction industry: BIM as a construction data digitalisation tool, IoT as a data collecting tool from construction components and Blockchain as data protection and records management. The three technologies strengthen each other's function and contribute to expected gains in time and resources efficiency [19]. 
Aleksandrova et al. (2019) presented a model of an ecosystem for the currently trending digitalization technologies functioning together in order to improve firms' management and control over projects and assets. The ecosystem includes BIM, Big Data, Blockchain, IoT, 3D printing, VR, AR and cloud computing. The authors consider BIM as the base of the digitalization pyramid in the construction industry, and other technologies such as Blockchain should be developed to integrate within the BIM environment. The proposed model suggests merging the BIM model with Blockchain in a cloud-based shared workspace. Implementing Blockchain and other integration technologies with BIM is expected to improve the decision-making process and to improve the quality of the management process [20].

According to Singh and Ashuri [21], the BIM design process transformed the design documentation from stamped paper designs that are shared with stakeholders to BIM models that hold elements, history and dependencies and that are meant to be used in a collaborative workflow. However, the adoption of BIM introduces a different type of issues such as litigations, data standardization, and responsibility for the produced design, copyright use and management. The authors see a solution for these issues in the emerging Blockchain technology and present a framework that is supported by a Blockchain for construction design events, based on real-time captured data. The authors expect that such a framework will improve data transparency, traceability and integrity. In addition, the collected data could be utilized for other purposes, such as machine learning and artificial intelligence applications.

\subsection{Blockchain and Construction Data}

Data protected by Blockchain is viewable and accepts adding as a new version without tampering with previous versions, and it is therefore considered immutable. Such features change the trust question from do you trust this person? To do you trust this system? [22].

Cyber threats in construction companies are often misunderstood and underestimated, which leads to the adoption of inadequate protocols and tools [23]. The NBS defines Common Data Environment (CDE) as "a central repository where construction project information is hosted [24]. The contents of the CDE are not limited to assets created in a 'BIM environment,' and will, therefore, include documentation, graphical model and non-graphical assets.". Current $\mathrm{CDE}$ is hosted on the web and is accessible using a Uniform Resource Locator (URL), which is exposed to cyberattacks [23]. PAS 1192-5:2015 directed toward BIM users, providing measures to avoid cyber-attacks [25]. However, $\mathrm{CDE}$ includes more than BIM. Hence, more comprehensive guide is required to address the problem properly [23]. The new ISO 19650-1/2:2018 provided an improved and a comprehensive view to manage digital construction works [26]. Parn and Edwards [23] suggest using Blockchain to serve as a peer-to-peer network to exchange construction data. The proposed framework suggested sharing digital assets after converting them to a block, and then broadcasting it to the CDE federated Blockchain network. After accepting the new asset block on the network, it becomes immutable and accessible only by authorised nodes.

Zheng et al. (2019) discussed roles and privileges for accessing mobile cloud BIM with Blockchain enhancements. BIM data protection faces three main challenges: (1) data is accessible anywhere, anytime for authorised participants; (2) users roles and privileges overlap which makes the traditional Role-Based Access Control (RBAC) inefficient; (3) risk of intentional or unintentional credential leak [27]. To tackle these challenges, Zheng et al. [27] propose a smart context-aware user and privileges management system called Context-aware Access Control mechanism (CaAC). The authors claim that RBAC, the mainstream system used today in BIM-related applications, lacks flexibility in roles and privileges definitions: the user either has access or does not without considering context. The CaAC system aims to control BIM by considering the environment around the requested permission, such as location and time. Five factors are considered in CaAC: (1) users; (2) roles; (3) objects; (4) operation; (5) permissions. CaAC separates users from roles and uses many to many relationships to allow each user to have more than one role, and each role to be assigned to more than one user. The CaAC system depends on the technological improvement of other technologies such as Blockchain, AR, VR, GPS, IoT and AI for data control and context recognition.

\subsection{Blockchain and Construction Supply Chain}

Fitriawijaya et al. [28] developed a model to integrate Supply Chain Management (SCM) with BIM to create decentralised CDE based on Blockchain technology. The reason why improving the supply chain is essential to construction projects are that two elements of the project management constraint triangle are connected directly to the supply chain: cost and time [29]. Fitriawijaya et al. [28] claim that a Blockchain-based SCM is transparent, traceable and efficient because the technology solves the mistrust issue and reduces transaction costs. The authors suggest the use of smart contracts as a transaction management tool to handle the trading process, which will simplify the relationship between participants in what is called a Blockchain trustless verification.

Sivula et al. (2018) claim that successful Blockchain implementation in the construction supply chain would require cooperative behaviour from the supply chain members. Two challenges and obstacles facing Blockchain adoption in the supply chain industry are: (1) the nature of the industry itself: supply chains, in general, contain a vast number of actors, multiple production points and multiple delivery locations; (2) sharing local companies working 
protocols: giving access for customers to track their orders means sharing internal working procedures which could be misinterpreted. Identified opportunities for supply chain based Blockchain are (1) records for all lifecycle customers from contractor to end-user; (2) efficient payment system; (3) transparency; (4) one database that contains all the information [30].

Wang [31] present a pilot study that examines Blockchain when used to manage the construction supply chain. The study followed the activities of a consortium of British construction companies and was divided into two parts, a pre-pilot stage and a pilot stage. The pre-pilot study used the sense-making process based on the theory of sensemaking, which provides tools to examine organisational actors' cognition and situated actions when introduced to a new technology [32]. The pilot stage study was conducted as a qualitative study. The researchers attended work meetings, conducted interviews and organised focus groups. Findings from the study include: (1) current business models require deep changes in order to adopt Blockchain successfully; (2) Blockchain technology's capability to provide a decentralized solution should not necessarily be perceived as a decentralized decision-making process, especially in cumulative decision-making processes such as supply chain management.

Kifokeris and Koch [33] reviewed the available literature about Blockchain in Construction, especially the publications connected to actors and workflow in construction logistics. The study identifies three groups of buildings logistics actors as main beneficiaries from Blockchain if it is adopted within the industry: (1) large contractors to improve their control and overcome obstacles during the supply process; (2) clients who used thirdparty suppliers in order to reduce uncertainty and improve the trust among different parties; (3) third party actors who are considered dedicated building logistics services providers for example equipment leasing companies. The authors criticize the current literature, concluding that "The largely non-systematic approach in most of these potential implementation efforts reveals that they are still rather visions than actual applications. Therefore, researchers should commence an analysis of possible issues related to the embedding and interaction of blockchain within building process and practices".

\subsection{Blockchain and Construction Concrete Quality}

Concrete is an essential element of the construction industry. Bohner et al. (2019) investigated digitalising the concrete quality control process in Finland. The authors describe the current quality management procedure as being document-based through exchanging emails, which is inefficient because the data collected is not ready for analysis without further processing. They propose an alternative system that links the concrete quality data with its corresponding element in BIM models, and by replacing the documents with CDE compatible reports. The system uses Blockchain to ensure data integrity because stakeholders do not share the same interest from the collected data, and the expected concrete serviceability period is considerable. Therefore, data should be distributed among all participants and be accessible even if in the future, one of the participants does not exist anymore. For feasibility reasons, the proposed system suggests storing fingerprint data only which can be used to verify data stored outside the Blockchain [34].

Lanko et al. [35] presented a combination of RFID and Blockchain to address the trust issue between parties, in addition to product safety during transportation. Currently, concrete quality is determined after concrete pouring with tests; The authors propose an alternative solution using Blockchain and RFID, that responds to the mistrust issue when dealing with concrete producers by using RFID tags in each step inside the factory and broadcasting this data as timestamped transactions to a Blockchain database. This will make the production process transparent and trackable if combined with GPS technology.

\subsection{Blockchain and Construction Corruption and Permits Compliance}

Nowadays, BIM is seen as an interface for the whole transformation of the construction industry that improves and facilitates collaboration by simplifying the data exchange processes [36]. One of these processes is compliance checking. Compliance checking is part of the pre-construction phase, where the building design data is submitted to the regulating authorities to check if the design aligns with the building codes. Such a process is time-consuming and challenging for the authorities. Nawari and Ravindran [36] propose a framework to automate this process using a combination of Blockchain and BIM. The proposed system requires building a classification supported with definitions, then converting the rules from law and regulation to logical programmable codes. The second step is executing these rules, which will lead to approve the design to be built or return it to their owner for modifications. Such a data-sensitive process should be performed under a secured and collaborative environment to save the client and authorities. These needs can be satisfied if Blockchain is used as data processing and protection technology.

Construction processes can be especially inefficient in post-disaster recovery scenarios when decisions are made under increased pressure [37]. The need to rush to fix the disaster's effect on society is a chance for fraud and malicious activities by suspicious parties because authorities in the post-disaster situation are fragmented and uncoordinated [37]. These authors propose automating the building permitting process using Blockchain to develop an 
Automated Code-checking and Compliance (ACCC) system. Currently, the clash detection feature in BIM authoring software presents a good start; however, it is limited and does not cover all compliance areas such as local regulations and environmental conditions [37]. Automating or semi-automating the building permitting process will save time which is a crucial factor in the post-disaster process. The ACCC framework proposed by Nawari is based on logical rule-checking principles and contains three primary components: (1) Generalized Adaptive Framework (GAF) which was built on Dimyadi's work [38] to automate compliance audit process for building information models; (2) smart contracts; (3) Blockchain network systems. Besides a speedier permitting process, the authors expect this framework will provide additional benefits such as storing all the permitting documents in one immutable record and one location to access all data related.

Ablyazov and Petrov [39] suggest using Blockchain to build an interaction system between stakeholders to reduce risk and unpredictability when investing in a construction project. For example, BitRent developed by Dmitriy Starovoitov [40] is a digital platform that is designed to help investors monitor work progress using two subprocesses: (1) Open Modeling (OM), which allows the investor to view and track the design process; (2) Automated Progress Monitoring (APM), by including RFIDs in the construction elements and keep its status updated on the platform [39].

Li et al. [41] argue that smart government based on Blockchain would provide new possibilities to provide services in areas such as tax collection, properties, and land registration. Challenges for implementing Blockchain according to the author are (1) ensuring data connectivity; (2) coding smart contracts; (3) energy consumption; (4) instability of crypto-currency prices; (5) interoperability; (6) legal limitations; (7) cyber-attacks; (8) change-resistance culture; (9) the insufficient level of digitalisation in the industry. However, opportunities also are expected for implementing Blockchain, such as (1) improved collaboration; (2) development of digital twins; (3) removing intermediates; (4) process facilitation; (5) ownership traceability; (6) real-time components tracking; (7) human errors minimisations; (8) smart contracts; (9) audibility; (10) improved open workflow.

\subsection{Blockchain and Smart Cities}

Li (2018) [42] identify three underlying technologies for smart cities using IoT and cloud computing: (1) Big data including traffic, environmental and metrological data; (2) Energy Internet to transform legacy power systems into flexible, adaptive systems [43]; (3) IoT as a data collector and action executer [42]. This author considers Blockchain as a solution for problems which are introduced by these technologies, such as data credibility and privacy in IoT and energy internet in addition to centralised storage costs and data preservation against attacks for big data from human attacks or natural disasters.

The Dynamic Alliance (DA) based on Blockchain technology concept was used by Zhang et al. [44] as a solution for smart cities resources allocation. Zhang et al. [44] expect that DA-Blockchains save cities costs and time by improving information exchange workflows. Qian et al. [45] consider smart cities as large data-sharing platforms between the society participants. The relationship between these participants is described as trustless, which is expected when a traditional data exchange system is used to manage sensitive information [45]. The issue with smart cities data is that it could be used for more than one purpose and Qian et al. [45] suggest using Blockchain to control its use. The proposed framework stores data into three levels: (1) a public data directory where metadata is stored; (2) smart contracts which have access to the data directory and the private data to verify conditions before execution; (3) data operation record where actions such as encrypted data requests and contracts execution are stored [45].

El-Diraby et al. [46] consider that controlling cities using the control-room approach is inefficient and unsustainable because it does not respond to the challenges smart cities are facing in addition to considering citizens of the city as information receivers only instead of treating them as knowledge sources and innovators. Challenges for data in smart cities include (1) complexity for example priorities for urban-related decisions; (2) multidisciplinarity as cities face problems from several infrastructural sources; (3) subjectivity where each problem should be analysed according to its context to have an effective solution [46]. These challenges could benefit from citizens' empowerment to create better knowledge and reflect it on the decisions made. El-Diraby et al. [46] suggest using a Blockchainpowered feedback system where the data becomes accessible for citizens from its source (such as IoT sensors), then make it possible for them to participate in the decision making process.

Klyukin et al. (2018) investigated the expected effect of implementing Blockchain in the urban planning process. Urban planning information is sensitive as it contains cities' future development plans. Leaking data to one party and hiding it from another leads to an advantage for the informed party. However, data exchange is necessary for certain parties such as legislators for discussion and approval before becoming effective. Urban plans should also be accessible for all citizens to help them plan their life according to the city plans and participate in the decision-making process. Blockchain provides a new method for interaction between citizens, commercial and legal entities while ensuring data safety and integrity. Expected benefits of using Blockchain in urban planning are: (1) reduction of errors of documents circulation; (2) increased urban design quality as a result of enhanced interactivity; (3) improved data accessibility; (4) transparency; (5) accelerated regulation process; (6) improved resources allocation; (7) permits 
traceability; (8) track land use and abuse; (9) remove intermediaries; (10) increased citizen-awareness as they become involved in the process [47].

\subsection{Blockchain and Civil Engineering Processes}

Lam et al. (2018) consider that Blockchain is suitable for civil engineering practices in (1) site investigation works at the beginning of the project such as surveying; (2) design and tender stage signatures, design update and permits records; (3) site activities including safety records; (4) excavation work process including dumping time and location; (5) material quality controls including testing records. In addition to Blockchain, technologies like IoT and RFID are essential to automate the collecting data process [48].

Jo et al. (2018) combine IoT with Blockchain to make SHM processes efficient and secured. Currently, SHM systems are centralised and stored on a local server or in the cloud. This is not practical because the number of connected devices is growing, which will require increased computing power and more abundant storage in addition to the security concerns related to the collected data. IoT for SHM involves installing sensors on a structure to make sure it is healthy and not causing safety concerns for society. However, parties involved in the process, such as the monitoring team, client, and maintenance team, have different priorities which make the relationship among them trustless. Besides the trust issue, the data confidentiality for the structure is a primary concern. The authors believe that a combination of Blockchain with IoT can be adopted to provide reliable information besides solving the single point of failure in centralised storage systems [49]. The solution proposed by Jo et al. (2018) is built on a consortium Blockchain to provide decentralised, reliable data storage and uses smart contracts to enhance safety by triggering alerts automatically when the data gathered from the sensors is abnormal. However, because the SHM data collected from the sensors itself is meaningless unless interpreted according to its sources and location, the proposed system does not encrypt it in the Blockchain but encrypts its interpretation. Such data could be used in litigations if the law started to consider it a legally acceptable source of information. On the other hand, the proposed system requires a minimum number of active nodes to verify new transactions and the real-time feature requires faster time for block production which reduces the strength of the Blockchain technology [49].

\section{Conclusions}

This paper aims to investigate Blockchain applications in the construction industry bibliometrics and to analyse its content. The literature was collected from Web of Knowledge and Scopus. Considering that the topic is very recent, it was expected that the amount of literature would be limited and that the level of information inside would be mainly at a conceptual level. Most of the literature starts with a section about Blockchain technology to explain its fundamentals, types, and capabilities. Later sections explain the possible applications and the expected results.

Bibliometrics show that Nawari and Ravindran are the most productive authors. The most productive country was China, with four articles, and Turk is the most cited author. A total of 34 articles were analysed. The literature was divided into three categories according to the best fit based on their content: (1) construction management; (2) building information modelling; (3) civil work and infrastructure.

Category 1 separates the construction industry need for Blockchain from other industries. Several applications were identified such as (1) smart contracts; (2) supply chain management and circular economy; (3) Building Information Modelling (BIM); (4) facility management; (5) sustainability. Benefits from Blockchain were identified such as (1) documents ownership; (2) copyright protection; (3) modifications and errors responsibilities. Blockchain in construction faces a tough challenge, changing the dominant culture of human-based decisions to automated decisions. Such challenge can be solved with gradual technology adoption and raising awareness of the advantages of the technology.

Category 2 focuses on BIM and its application with Blockchain. However, a combination with other technologies like RFID and IoT show a strong presence. BIM's current problems, such as data protection, rules and privileges, and automated compliance, were also discussed.

Category 3 discussed the literature about Blockchain and civil work and infrastructure. SHM based Blockchain, and concrete quality was presented as applications for Blockchain to improve civil engineering practices. In smart cities' application, Blockchain was presented as a solution to improve current urban planning processes, empower citizens, and to change the way cities deal with collected data.

The main intended contributions of this paper are the following: (1) To identify existing initiatives to implement Blockchain in the Construction Industry; (2) to provide a forecast for the technology growth directions based on the currently available bibliometrics. There is a considerable agreement between researchers about the future role for Blockchain in improving construction collaboration and construction data integrity. 
The main limitations of this study are due to the small volume of literature found from the main indexing databases, which may reduce the significance of the bibliometrics. Indeed, as is typical of recent research fields, the greater part of the available literature was composed of conference articles where early findings are presented.

As an emerging topic, the room for future reviews in the domain of Blockchain in Construction is vast. The authors expect future reviews to focus on Blockchain applications from case studies as these become available. Despite the small volume of available literature, an assessment of the recent evolution in research indicators in the area of Building and Construction, combined with that of other industrial activities where Blockchain adoption is more mature, suggests a promising future for this research field.

\section{Conflicts of Interest}

The authors declare no conflict of interest.

\section{References}

[1] Crosby, Michael, Pradan Pattanayak, Sanjeev Verma, and Vignesh Kalyanaraman. "Blockchain technology: Beyond bitcoin." Applied Innovation 2, no. 6-10 (2016): 71.

[2] Penzes, B., A. KirNup, C. Gage, T. Dravai, and M. Colmer. "Blockchain Technology in the Construction Industry: Digital Transformation for High Productivity." Institution of Civil Engineers (ICE), (2018).

[3] Aria, Massimo, and Corrado Cuccurullo. "Bibliometrix : An R-Tool for Comprehensive Science Mapping Analysis." Journal of Informetrics 11, no. 4 (November 2017): 959-975. doi:10.1016/j.joi.2017.08.007.

[4] Turk, Žiga, and Robert Klinc. "Potentials of Blockchain Technology for Construction Management.” Procedia Engineering 196 (2017): 638-645. doi:10.1016/j.proeng.2017.08.052.

[5] Hardin, Brad, and Dave McCool. BIM and construction management: proven tools, methods, and workflows. John Wiley \& Sons, (2015).

[6] Wang, Jun, Peng Wu, Xiangyu Wang, and Wenchi Shou. "The Outlook of Blockchain Technology for Construction Engineering Management.” Frontiers of Engineering Management 4, no. 1 (2017): 67. doi:10.15302/j-fem-2017006.

[7] Shojaei, Alireza. "Exploring Applications of Blockchain Technology in the Construction Industry." Edited by Didem Ozevin, Hossein Ataei, Mehdi Modares, Asli Pelin Gurgun, Siamak Yazdani, and Amarjit Singh. Proceedings of International Structural Engineering and Construction 6, no. 1 (May 2019). doi:10.14455/isec.res.2019.78.

[8] Szabo, Nick. "Smart Contracts (1994)." Virtual School (1994).

[9] San, Kiu Mee, Chia Fah Choy, and Wong Phui Fung. "The Potentials and Impacts of Blockchain Technology in Construction Industry: A Literature Review.” IOP Conference Series: Materials Science and Engineering 495 (June 7, 2019 ): 012005. doi:10.1088/1757-899x/495/1/012005.

[10] Hargaden, Vincent, Nikolaos Papakostas, Anthony Newell, Adam Khavia, and Adam Scanlon. "The Role of Blockchain Technologies in Construction Engineering Project Management.” 2019 IEEE International Conference on Engineering, Technology and Innovation (ICE/ITMC) (June 2019). doi:10.1109/ice.2019.8792582.

[11] Luo, Han, Moumita Das, Jun Wang, and Jack C.P. Cheng. "Construction Payment Automation through Smart Contract-Based Blockchain Framework." Proceedings of the 36th International Symposium on Automation and Robotics in Construction (ISARC) (May 24, 2019). doi:10.22260/isarc2019/0168.

[12] K. C. Goh, T. Y. Teoh, H. H. Goh, K. Bilal, and C. S. Chai, "Blockchain potentials in enhancing construction stakeholders collaboration, Malaysian Construction Research Journal, Article vol. 7, no. Special issue 2, pp. 177-189, 2019.

[13] S. Graham and J. D. Hailer, "Implementation of Blockchain Technology in the Construction Industry" in Proceedings, Annual Conference - Canadian Society for Civil Engineering, (June 2019).

[14] Safa, Mahdi, Sylvia Baeza, and Kelly Weeks. "Incorporating Blockchain Technology in Construction Management." Strategic Direction 35, no. 10 (October 14, 2019): 1-3. doi:10.1108/sd-03-2019-0062.

[15] Nawari, Nawari O., and Shriraam Ravindran. "Blockchain technology and BIM process: Review and potential applications." ITcon 24 (2019): 209-238.

[16] Liu, Jiang, Osmani, and Demian. "Building Information Management (BIM) and Blockchain (BC) for Sustainable Building Design Information Management Framework.” Electronics 8, no. 7 (June 26, 2019): 724. doi:10.3390/electronics8070724.

[17] Zheng, Rongyue, Jianlin Jiang, Xiaohan Hao, Wei Ren, Feng Xiong, and Yi Ren. "bcBIM: A Blockchain-Based Big Data Model for BIM Modification Audit and Provenance in Mobile Cloud." Mathematical Problems in Engineering 2019 (March 18, 2019): 1-13. doi:10.1155/2019/5349538. 
[18] Bukunova, O.V., and A.S. Bukunov. "Tools of Data Transmission at Building Information Modeling." 2019 International Science and Technology Conference "EastConf" (March 2019). doi:10.1109/eastconf.2019.8725373.

[19] Ye, Zihao, Mengtian Yin, Llewellyn Tang, and Haobo Jiang. "Cup-of-Water Theory: A Review on the Interaction of BIM, IoT and Blockchain during the Whole Building Lifecycle." Proceedings of the 35th International Symposium on Automation and Robotics in Construction (ISARC) (July 22, 2018). doi:10.22260/isarc2018/0066.

[20] Aleksandrova, Elena, Victoria Vinogradova, and Galina Tokunova. "Integration of Digital Technologies in the Field of Construction in the Russian Federation.” Engineering Management in Production and Services 11, no. 3 (November 19, 2019): 38-47. doi:10.2478/emj-2019-0019.

[21] Singh, Sulekha, and Baabak Ashuri. "Leveraging Blockchain Technology in AEC Industry during Design Development Phase.” Computing in Civil Engineering 2019 (June 13, 2019). doi:10.1061/9780784482421.050.

[22] Dakhli, Zakaria, Zoubeir Lafhaj, and Alan Mossman. “The Potential of Blockchain in Building Construction.” Buildings 9, no. 4 (April 5, 2019): 77. doi:10.3390/buildings9040077.

[23] Parn, Erika A., and David Edwards. "Cyber Threats Confronting the Digital Built Environment." Engineering, Construction and Architectural Management 26, no. 2 (March 18, 2019): 245-266. doi:10.1108/ecam-03-2018-0101.

[24] McPartland, R. "What is the Common Data Environment (CDE)." Corporate Website of NBS. (2016). Available online: https://www.thenbs.com/knowledge/what-is-the-common-data-environment-cde.

[25] BSI, SC. "PAS 1192-5: 2015: Specification for security-minded building information modelling, digital built environments and smart asset management." (2015). Available online: https://shop.bsigroup.com/ProductDetail/?pid=000000000030314119

[26] ISO, BSEN. "19650-1: 2018." BSI Standards Publication Organization and digitization of information about buildings and civil engineering works, including building information modelling (BIM)-Information management using building information modelling (2018).

[27] Zheng, Rongyue, Jianlin Jiang, Xiaohan Hao, Wei Ren, Feng Xiong, and Tianqing Zhu. "CaACBIM: A Context-Aware Access Control Model for BIM.” Information 10, no. 2 (February 1, 2019): 47. doi:10.3390/info10020047.

[28] Fitriawijaya, Adam, and Tsai Hsin-Hsuan. "A Blockchain Approach to Supply Chain Management in a BIM-Enabled Environment." (2019).

[29] Van Wyngaard, C. J., J. H. C. Pretorius, and L. Pretorius. "Theory of the Triple Constraint \&\#x2014; A Conceptual Review." 2012 IEEE International Conference on Industrial Engineering and Engineering Management (December 2012). doi:10.1109/ieem.2012.6838095.

[30] Sivula, Ari, Ahm Shamsuzzoha, and Petri Helo. "Blockchain in Logistics: Mapping the Opportunities in Con-struction Industry." In International Conference on Industrial Engineering and Operations Management. (2018).

[31] Wang, Yingli. "Designing a Blockchain Enabled Supply Chain.” IFAC-PapersOnLine 52, no. 13 (2019): 6-11. doi:10.1016/j.ifacol.2019.11.082.

[32] Jensen, Tina Blegind, Annemette Kjærgaard, and Per Svejvig. "Using Institutional Theory with Sensemaking Theory: A Case Study of Information System Implementation in Healthcare.” Journal of Information Technology 24, no. 4 (December 2009): 343-353. doi:10.1057/jit.2009.11.

[33] Kifokeris, Dimosthenis, and Christian Koch. "Blockchain in Construction Logistics: State-of-Art, Constructability, and the Advent of a New Digital Business Model in Sweden.” Proceedings of the 2019 European Conference on Computing in Construction (July 10, 2019). doi:10.35490/ec3.2019.163.

[34] Bohner, Edgar, Tapio Vehmas, Arto Laikari, Matti Okkonen, Markku Kiviniemi, Pertti Lahdenperä, and Miguel Ferreira. "Digitalisation of the quality control of concrete manufacturing and construction." In fib Symposium 2019: ConcreteInnovations in Materials, Design and Structures. International Federation for Structural Concrete FIB, (2019): 1435-1442.

[35] Lanko, A., N. Vatin, and A. Kaklauskas. "Application of RFID Combined with Blockchain Technology in Logistics of Construction Materials." Edited by I. Ilin and O. Kalinina. MATEC Web of Conferences 170 (2018): 03032. doi:10.1051/matecconf/201817003032.

[36] Nawari, Nawari O., and Shriraam Ravindran. "Blockchain and the Built Environment: Potentials and Limitations." Journal of Building Engineering 25 (September 2019): 100832. doi:10.1016/j.jobe.2019.100832.

[37] Nawari, Nawari O., and Shriraam Ravindran. "Blockchain and Building Information Modeling (BIM): Review and Applications in Post-Disaster Recovery.” Buildings 9, no. 6 (June 19, 2019): 149. doi:10.3390/buildings9060149.

[38] Dimyadi, Johannes. "Automated Compliance Audit Processes for Building Information Models with an application to performance-based fire engineering design methods." PhD diss., ResearchSpace@ Auckland, (2016). 
[39] Ablyazov, Timur, and Ivan Petrov. "Influence of Blockchain on Development of Interaction System of Investment and Construction Activity Participants.” IOP Conference Series: Materials Science and Engineering 497 (April 2, 2019 ): 012001. doi:10.1088/1757-899x/497/1/012001.

[40] Coinhills. "BitRent." @ Coinhills. Available online: https://www.coinhills.com/ico/view/bitrent/

[41] Li, Jennifer, David Greenwood, and Mohamad Kassem. "Blockchain in the Built Environment and Construction Industry: A Systematic Review, Conceptual Models and Practical Use Cases.” Automation in Construction 102 (June 2019): $288-307$. doi:10.1016/j.autcon.2019.02.005.

[42] Li, Shuling. "Application of Blockchain Technology in Smart City Infrastructure." 2018 IEEE International Conference on Smart Internet of Things (SmartIoT) (August 2018). doi:10.1109/smartiot.2018.00056.

[43] Dong, Zhaoyang, Junhua Zhao, Fushuan Wen, and Yusheng Xue. "From smart grid to energy internet: basic concept and research framework." Automation of electric power systems 38, no. 15 (2014): 1-11.

[44] Zhang, Yi, Wei Sun, and Chenlei Xie. "Blockchain in Smart City Development-The Knowledge Governance Framework in Dynamic Alliance.” Advancements in Smart City and Intelligent Building (2019): 137-152. doi:10.1007/978-981-13-67335_13.

[45] Qian, Yuming, Zhicheng Liu, Junyan Yang, and Qiao Wang. "A Method of Exchanging Data in Smart City by Blockchain." 2018 IEEE 20th International Conference on High Performance Computing and Communications; IEEE 16th International Conference on Smart City; IEEE 4th International Conference on Data Science and Systems (HPCC/SmartCity/DSS) (June 2018). doi:10.1109/hpcc/smartcity/dss.2018.00223.

[46] El-Diraby, Tamer, Alain Zarli, and Mohamed El-Darieby. "The Automation of the Softer Side of Smart City: a SocioSemantic Roadmap." Proceedings of the 36th International Symposium on Automation and Robotics in Construction (ISARC) (May 24, 2019). doi:10.22260/isarc2019/0035.

[47] Klyukin, Aleksei A., Valerii N. Kulachkovsky, Valerii N. Evseev, and Anna I. Klyukina. "Possibilities of New Information Technologies in the System of Urban Planning and Construction.” Key Engineering Materials 771 (June 2018): 49-55. doi:10.4028/www.scientific.net/kem.771.49.

[48] Lam, R.C.Y., Alvin Junus, J.Y.W. Mak, L.C.H. Lam, and P.K.K. Lee. "Blockchain for Civil Engineering Practices in Smart Cities." 2018 IEEE International Conference on Internet of Things (iThings) and IEEE Green Computing and Communications (GreenCom) and IEEE Cyber, Physical and Social Computing (CPSCom) and IEEE Smart Data (SmartData) (July 2018). doi:10.1109/cybermatics_2018.2018.00225.

[49] Jo, Byung, Rana Khan, and Yun-Sung Lee. "Hybrid Blockchain and Internet-of-Things Network for Underground Structure Health Monitoring." Sensors 18, no. 12 (December 4, 2018): 4268. doi:10.3390/s18124268. 